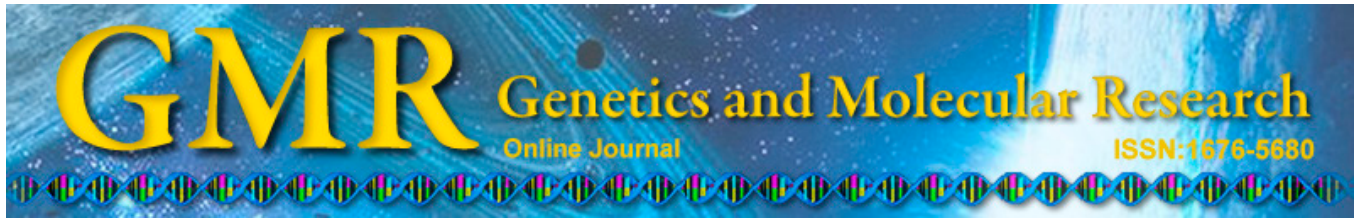

\title{
Effect of Bifidobacterium on the mRNA expression levels of TRAF6, GSK-3 $\beta$, and microRNA-146a in LPS-stimulated rat intestinal epithelial cells
}

W. Zhou, Y. Yuan, J. Li, W.M. Yuan, L.G. Huang and S.W. Zheng

Department of Neonatology, Guangzhou Women and Children's Medical Center, Guangzhou Medical University, Guangzhou, Guangdong Province, China

Corresponding author: W. Zhou

E-mail: zhouweils@yeah.net

Genet. Mol. Res. 14 (3): 10050-10056 (2015)

Received December 12, 2014

Accepted June 23, 2015

Published August 21, 2015

DOI http://dx.doi.org/10.4238/2015.August.21.11

\begin{abstract}
We investigated the effect of inactivated Bifidobacterium on the mRNA expression of TRAF6, GSK-3 $\beta$, and microRNA-146a in lipopolysaccharide (LPS)-stimulated rat small intestinal epithelial cells (IEC-6s). IEC-6s were randomly divided into an LPS group, a culture supernatant group, and an inactivated bacteria group. After stimulation with LPS for $5 \mathrm{~h}$, the three groups were treated as follows: the LPS group was cultured for $24 \mathrm{~h}$ with sterile saline; the culture supernatant group was cultured with Bifidobacterium (infantis strain) culture supernatant for $24 \mathrm{~h}$; and the inactivated bacteria group was cultured with inactivated infantis Bifidobacterium for $24 \mathrm{~h}$. Reverse transcription polymerase chain reaction was used to determine mRNA expression levels. The mRNA expression levels of TRAF-6 and GSK$3 \beta$ in the culture supernatant group were lower, and microRNA-146a expression was higher, compared with the LPS group $(t=5.278, \mathrm{P}=$ $0.000 ; t=6.316, \mathrm{P}=0.000 ; t=13.218, \mathrm{P}=0.000$, respectively). GSK-
\end{abstract}


$3 \beta$ mRNA expression in the inactivated bacteria group was lower than in the LPS group $(t=4.837, \mathrm{P}=0.000)$. There was no difference in the mRNA expression levels of TRAF- 6 and microRNA-146a between the two groups $(t=0.732, \mathrm{P}=0.472$ and $t=1.463, \mathrm{P}=0.164)$. Both the culture supernatant and the inactivated Bifidobacterium had a protective effect on LPS-stimulated IEC-6s. The protective effect of Bifidobacterium may be achieved through increased microRNA-146a by reducing levels of TRAF6 and GSK-3 $\beta$; the protective effect of inactivated Bifidobacterium may be achieved by reducing levels of GSK-3 $\beta$.

Key words: Intestinal epithelial cells; Rat; Bifidobacterium; TRAF6; GSK-3ß; microRNA-146a

\section{INTRODUCTION}

Neonatal necrotizing enterocolitis (NEC) is an acute necrotizing intestinal disease caused by multiple perinatal pathogenic factors. It mainly occurs in premature children, especially in children of extremely low birth weight, and the fatality rate can be as high as $50 \%$. Currently, fasting and gastrointestinal decompression are mainly used for the early treatment of NEC. Recent meta-analyses have shown that for high-risk preterm children, prophylactic use of probiotics (Lactobacillus and Bifidobacterium) can reduce the incidence of NEC and mitigate the severity of the disease (Lin et al., 2005, 2008). Studies have shown that Bifidobacterium and its culture supernatant may have a beneficial effect on intestinal cells, but the mechanisms by which they act are not identical (McElroy et al., 2012; Bermudez-Brito et al., 2013). In this study, we treated rat small intestinal epithelial cells (IEC-6s) that had been co-cultured with lipopolysaccharides (LPS) with inactivated Bifidobacterium or Bifidobacterium culture supernatant, to explore the effect of Bifidobacterium on LPSstimulated IEC-6 and to provide experimental evidence for the application of Bifidobacterium in NEC prevention.

\section{MATERIAL AND METHODS}

\section{Materials}

IEC-6s were purchased from the Cell Resource Center, The Institute of Basic Medical Sciences (IBMS) of the Chinese Academy of Medical Sciences (CAMS) of the Peking Union Medical College (PUMC); Bifidobacterium (infantis strain) lyophilized powder, number GIM 1.207, was purchased from the Microbial Culture Collection Center of the Guangdong Institute of Microbiology (GIMCC); Dulbecco's modified Eagle's medium and trypsin were purchased from GIBCO (Paisley, United Kingdom); fetal bovine serum and phosphate-buffered saline were purchased from HyClone (Irvine, CA, USA); LPS (Escherichia coli 0127: B8) and bovine insulin were purchased from Sigma (St Louis, MO, USA); and a PrimeScript reverse transcription polymerase chain reaction (RT-PCR) kit (DRR036A), a One Step PrimeScript microRNA (miRNA) cDNA Synthesis Kit (D350A), and a SYBR Premix Ex Taq ${ }^{\mathrm{TM}}$ II (DRR081A) were purchased from TaKaRa. 


\section{Methods}

\section{Preparation of Bifidobacterium culture supernatant}

According to the method of Bermudez-Brito et al. (2013), infantis Bifidobacterium was anaerobically cultured in liquid medium for $48-72 \mathrm{~h}$ and centrifuged at $12,000 \mathrm{~g}$ and $4^{\circ} \mathrm{C}$ for 10 min. The supernatant was collected and $1 \mathrm{~N}$ sodium hydroxide solution was used to adjust the $\mathrm{pH}$ of supernatant to approximately 7.0. After filtration using a $0.22-\mu \mathrm{m}$ microporous membrane, the supernatant was stored at $-20^{\circ} \mathrm{C}$. It was returned to room temperature before use.

\section{Preparation of inactivated Bifidobacterium}

Referring to the method used by Xiong et al. (2005), the precipitate described in the previous section was placed in a water bath at $65^{\circ} \mathrm{C}$ for $30 \mathrm{~min}$ for inactivation (no viable bacteria presented in the liquid medium). The bacteria were washed and centrifuged with sterile saline at $4^{\circ} \mathrm{C}$ and 4000 revolutions per minute (rpm) several times, for $10 \mathrm{~min}$ each time. When the bacteria were white, their concentration was adjusted to $1 \times 10^{7}$ colony-forming units $(\mathrm{CFU}) / \mathrm{mL}$.

\section{Cell culture and grouping}

IEC-6 cells were incubated with Dulbecco's modified Eagle's medium containing 5\% fetal bovine serum and $0.01 \mathrm{~g} / \mathrm{L}$ insulin $\left(37^{\circ} \mathrm{C}, 5 \% \mathrm{CO}_{2}\right.$, humidified incubator). The medium was replaced every 1-2 days. When the fusion rate was $90 \%$, cells were treated with $0.25 \%$ trypsin and passaged at a ratio of 1:3; cells in the logarithmic phase were used for tests. The cells were randomly divided into an LPS group, a culture supernatant group, and an inactivated bacteria group. All cells were treated with $5 \mu \mathrm{g} / \mathrm{mL}$ LPS for $5 \mathrm{~h}$, then the medium was changed and $1 \mathrm{~mL}$ sterile saline, $1 \mathrm{~mL}$ Bifidobacterium culture supernatant, and $1 \times 10^{7} \mathrm{CFU} /$ $\mathrm{mL}$ inactivated Bifidobacterium were added for $24 \mathrm{~h}$.

\section{RT-PCR detection of the mRNA expression of TRAF-6, GSK-3B, and miRNA-146a}

Total RNA was extracted using the TRIzol method, and the concentration was adjusted to $1 \mu \mathrm{g} / \mu \mathrm{L}$. The reverse-transcribed cDNA was used for TRAF-6 and GSK-3 $\beta$ testing, according to the TaKaRa kit (DRR036A) manufacturer instructions. The reverse-transcribed cDNA was used for miRNA-146a testing, according to the TaKaRa kit (D350A) manufacturer instructions. Both RT-PCR detections were performed using the TaKaRa quantitative PCR kit, SYBR Premix Ex Taq ${ }^{\mathrm{TM}}$ II (DRR081A). The glyceraldehyde 3-phosphate dehydrogenase gene GAPDH was used as an internal reference for TRAF- 6 and GSK-3 $\beta$, and U6 was used as an internal control for miRNA-146a; samples in each group were analyzed using the relative quantitative analysis method ( $2^{-\Delta \Delta \mathrm{Ct}}$ method).

\section{Statistical analysis}

The SPSS 13.0 software was used for data analysis; the results are reported as means \pm standard deviation (means $\pm \mathrm{SD}$ ). Single factor variance analysis was used to compare the 
differences in expression levels between the TRAF-6, GSK-3 $\beta$, and miRNA-146a groups; P $<$ 0.05 was considered to be statistically significant.

\section{RESULTS}

The relative mRNA expression levels of TRAF- 6 and GSK-3 $\beta$ in the culture supernatant group were lower, and miRNA-146a expression was higher, compared with the LPS group, and the differences were statistically significant $(t=5.278, \mathrm{P}=0.000 ; t=6.316, \mathrm{P}=$ $0.000 ; t=13.218, \mathrm{P}=0.000)$. The relative mRNA expression levels of TRAF- 6 in the culture supernatant group were lower, and miRNA-146a expression was higher, compared with the inactivated bacteria group, and the differences were statistically significant $(t=6.009, \mathrm{P}=0.000$; $t=14.687, \mathrm{P}=0.000)$. There was a statistically significant difference in the relative mRNA expression of GSK-3 $\beta$ between the inactivated bacteria group and the LPS group $(t=4.837, \mathrm{P}$ $=0.000$ ); but there was no statistically significant difference in the relative mRNA expression levels of TRAF- 6 and miRNA-146a between the two groups $(t=0.732, \mathrm{P}=0.472 ; t=1.463$, $\mathrm{P}=0.164$ ), as shown in Table 1 and Figure 1 .

Table 1. Expression of TRAF-6, GSK-3 $\beta$, miRNA-146a mRNA in each group (means $\pm \mathrm{SD}, \mathrm{N}=6$ ).

\begin{tabular}{lccc}
\hline Group & TRAF-6 & GSK-3 & miRNA-146a \\
\hline LPS group & $1.01 \pm 0.14$ & $1.02 \pm 0.25$ & $1.01 \pm 0.12$ \\
Culture supernatant group & $0.72 \pm 0.05^{\mathrm{ab}}$ & $0.46 \pm 0.14^{\mathrm{a}}$ & $3.05 \pm 0.40^{\mathrm{ab}}$ \\
Inactivated bacteria group & $1.05 \pm 0.11$ & $0.59 \pm 0.13^{\mathrm{a}}$ & $0.78 \pm 0.22$ \\
F value & 21.499 & 21.831 & 130.802 \\
P value & 0.000 & 0.000 & 0.000 \\
\hline
\end{tabular}

${ }^{\mathrm{a}}$ compared with the lipopolysaccharide (LPS) group, $\mathrm{P}<0.01$; ${ }^{\mathrm{b}}$ compared with the inactivated bacteria group, $\mathrm{P}<$ 0.01 .

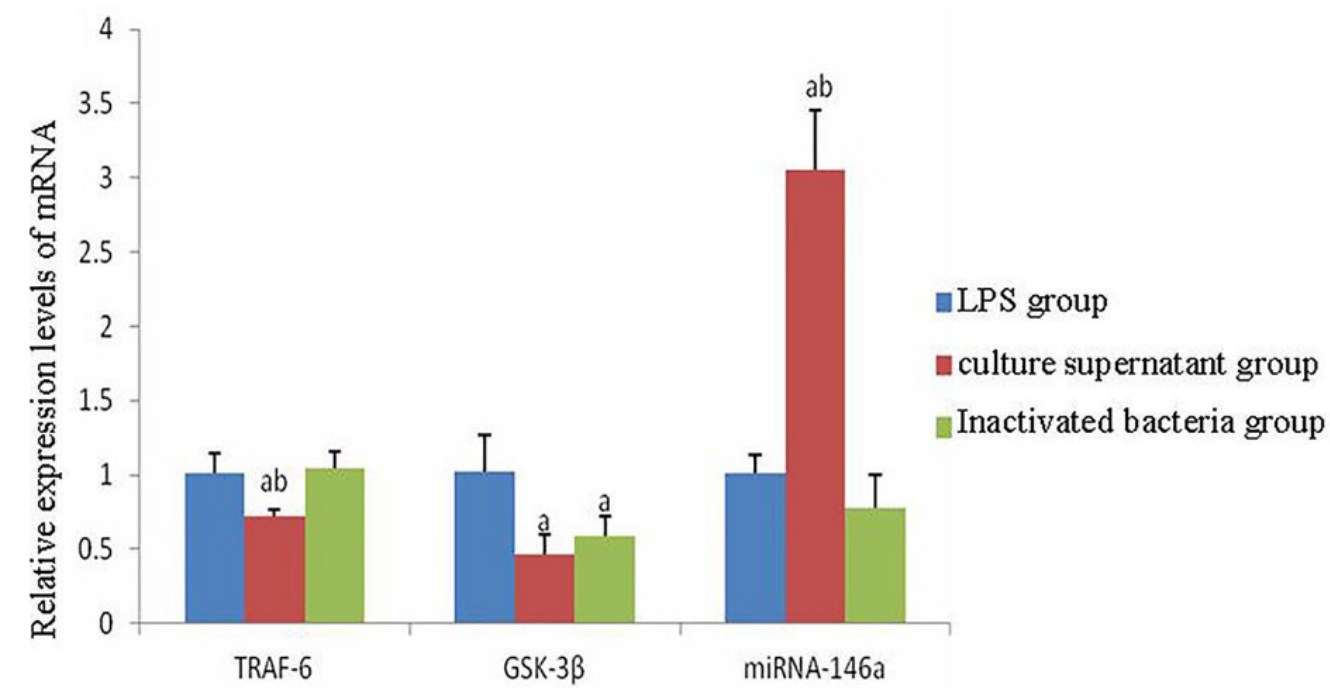

Figure 1. mRNA expression levels of TRAF-6, GSK-3 $\beta$, and microRNA-146a (miRNA-146a) in each group of cells. a: compared with the lipopolysaccharide (LPS) group, $\mathrm{P}<0.01$; b: compared with the inactivated bacteria group, $\mathrm{P}<0.01$. 


\section{DISCUSSION}

NEC can cause intestinal damage. The invasion of bacterial endotoxins causes an intestinal inflammatory cascade, ultimately resulting in intestinal epithelial cell necrosis. Clinical manifestations in children with the disease are mainly bloating, vomiting, diarrhea, and bloody stools, with shock and multiple organ failure in severe cases. Currently, fasting, total parenteral nutrition, intravenous broad-spectrum antibiotics, and other symptomatic therapies are the main methods for early treatment of the disease. In recent years, with a deepening understanding of the disease, researchers have found that probiotics can reduce the incidence, severity, and mortality of NEC (Ganguli and Walker, 2011; Li et al., 2013a). Studies have also shown that Bifidobacterium and their secretions are involved in intracellular signal transduction by regulating the tolllike receptor (TLR) signaling pathway to exert their biological functions.

The TLR signaling pathway mainly comprises TLRs and tumor necrosis factor receptor-associated factor 6 (TRAF-6). At present, 11 kinds of TLR are known. They include the following: TLR1-11, which is widely distributed in the B lymphocytes, dendritic cells, macrophages, endothelial cells, and epithelial cells; TLR-2, the ligands of which are the peptide glycans and lipoteichoic acid of the bacterial cells; and TLR-9, the ligand of which is nonmethylated CpG DNA (Bermudez-Brito et al., 2012). Moreover, research has shown that the TLR-9 signaling pathway is related to the anti-inflammatory effects of probiotics, which are considered to protect against NEC (Gribar et al., 2009; Bermudez-Brito et al., 2012). TRAF6 is an important member of the superfamily of tumor necrosis factors. Under physiological conditions it has a wide range of biological functions, playing an important role in neural tube closure, lymph node formation, and bone metabolism. In the TLR signal pathway, TRAF-6 can bind to phosphorylated IRAK-1, activating the NF- $\mathrm{KB}$ signaling pathway and generating a large amount of inflammatory mediators, finally causing inflammation (Doyle and O'Neill, 2006; Asagiri and Takayanagi, 2007). Therefore, it is possible to reduce inflammation to some extent by inhibiting the expression of TRAF-6. Some scholars have found that miRNA-146a can inhibit the function of TRAF-6 (Taganov et al., 2006).

MicroRNA (miRNA) is a class of non-coding RNA fragments, each comprising about 22 nucleotides (Nahid et al., 2011b). It can inhibit the expression of target mRNA by recognizing and binding the complementary sequences of the non-coding region at the $3^{\prime}$ end of the target mRNA (Rana, 2007; Chua et al., 2009). One study found that miRNA plays an important role in many aspects of cell biology, such as the regulation of cell proliferation, differentiation, and migration, and is associated with a variety of diseases (cancer, inflammation, and autoimmune diseases) (Baltimore et al., 2008; Lodish et al., 2008). Recent studies have found that miRNA, especially miRNA-146a, plays an important role in the regulation of the body's adaptive immunity and innate immunity systems (Lederhuber et al., 2011). Numerous studies have shown that various TLR ligands and inflammatory cytokines can promote the expression of miRNA-146a in an NF-kB-dependent manner; through a negative feedback mechanism, the overexpression of miRNA-146a leads to the reduced expression of TRAF- 6 (the adapter protein between NF- $\kappa B$ and TLRs), ultimately reducing the expression of IL- 6 , TNF- $\alpha$, and other cytokines in the downstream NF- $\mathrm{KB}$ signaling pathway, and thereby preventing the adverse consequences caused by the uncontrolled inflammatory response (Taganov et al., 2006; Nahid et al., 2011a).

Glycogen synthase kinase-3 (GSK-3) is the rate-limiting enzyme in glycogen synthesis. GSK- $3 \alpha$ and GSK-3 $\beta$ are the two main subtypes, and the latter has a more complex 
biological function (Wu and Pan, 2010). GSK-3 $\beta$ is closely associated to multiple signaling pathways. Recent studies have indicated that GSK-3 $\beta$ not only promotes the occurrence of inflammation, but also induces apoptosis and inhibits cell proliferation, ultimately leading to mucosal injury (Pang et al., 2009). Animal experiments have shown that reduced expression of GSK-3 $\beta$ can reduce intestinal damage caused by necrotizing enterocolitis in Wistar rats ( $\mathrm{Li}$ et al., 2013b). Sodhi et al. (2010) conducted in vivo and in vitro experiments and found that activated GSK-3 $\beta$ can inhibit the proliferation of intestinal epithelial cells by inhibiting the $\beta$-catenin signaling pathway. Studies have shown that TLR-2 and TLR-9 activation in macrophages may inhibit the expression of GSK-3 $\beta$, and then increase the expression of CREB, thereby reducing the inflammatory response (Martin et al., 2005).

The current study showed that mRNA expression of IEC-6 miRNA-146a in the supernatant group was significantly higher than in the LPS group, while mRNA expression of TRAF-6 and GSK-3 $\beta$ was downregulated compared with the LPS group. Bermudez-Brito et al. (2013) found that Bifidobacterium supernatant can upregulate TLR-9 in intestinal dendritic cells, thereby activating the TLR pathways to regulate the body's innate immune response and inflammation (Taganov et al., 2006). As with dendritic cells, IEC-6 can also express TLR-9. Recent studies have shown that Bifidobacterium can secret the TLR-9 ligand, unmethylated CpG DNA fragments (Bermudez-Brito et al., 2012, 2013). The above information and the results of this study suggest that the supernatant of Bifidobacterium may bind to TLR-9 in IEC-6 through a non-methylated CpG DNA fragment, activating the TLR-9 pathway of the IEC-6 cells and NF- $\mathrm{kB}$, and thereby causing the generation of miRNA-146a. miRNA-146a feedback inhibited TRAF- 6 expression, and in addition to TLR9 activation, mRNA expression of the GSK- $3 \beta$ was inhibited, thereby protecting the IEC-6 cells.

The study also showed that, compared with the LPS group, mRNA expression of GSK$3 \beta$ in IEC-6 of the inactivated Bifidobacterium group was significantly reduced. Bifidobacterium is a Gram-positive bacteria; the peptidoglycan in the cell wall is the TLR-2 ligand (BermudezBrito et al., 2012). Thus, it may be owing to the activation of TLR-2 in IEC-6 by peptidoglycan that the mRNA expression of GSK-3 $\beta$ was inhibited. However, why did inactivated Bifidobacterium not cause the generation of miRNA-146a as in the Bifidobacterium supernatants? The answer is probably that even in the same cell, the reaction generated by different TLRs may differ (Doyle and O'Neill, 2006). However, the specific mechanisms need to be explored further.

\section{Conflicts of interest}

The authors declare no conflict of interest.

\section{REFERENCES}

Asagiri M and Takayanagi H (2007). The molecular understanding of osteoclast differentiation. Bone 40: 251-264.

Baltimore D, Boldin MP, O’Connell RM, Rao DS, et al. (2008). MicroRNAs: new regulators of immune cell development and function. Nat. Immunol. 9: 839-845.

Bermudez-Brito M, Plaza-Díaz J, Muñoz-Quezada S, Gómez-Llorente C, et al. (2012). Probiotic mechanisms of action. Ann. Nutr. Metab. 61: 160-174.

Bermudez-Brito M, Muñoz-Quezada S, Gomez-Llorente C, Matencio E, et al. (2013). Cell-free culture supernatant of Bifidobacterium breve CNCM I-4035 decreases pro-inflammatory cytokines in human dendritic cells challenged with Salmonella typhi through TLR activation. PLoS One 8: e59370.

Chua JH, Armugam A and Jeyaseelan K (2009). MicroRNAs: biogenesis, function and applications. Curr. Opin. Mol. Ther. 11: 189-199. 
Doyle SL and O'Neill LA (2006). Toll-like receptors: from the discovery of NFkappaB to new insights into transcriptional regulations in innate immunity. Biochem. Pharmacol. 72: 1102-1113.

Ganguli K and Walker WA (2011). Probiotics in the prevention of necrotizing enterocolitis. J. Clin. Gastroenterol. 45: S133-S138.

Gribar SC, Sodhi CP, Richardson WM, Anand RJ, et al. (2009). Reciprocal expression and signaling of TLR4 and TLR9 in the pathogenesis and treatment of necrotizing enterocolitis. J. Immunol. 182: 636-646.

Lederhuber H, Baer K, Altiok I, Sadeghi K, et al. (2011). MicroRNA-146: tiny player in neonatal innate immunity. Neonatology 99: 51-56.

Li D, Rosito G and Slagle T (2013a). Probiotics for the prevention of necrotizing enterocolitis in neonates: an 8-year retrospective cohort study. J. Clin. Pharm. Ther. 38: 445-449.

Li ZY, Zhang BH, Tang R and Ling W (2013b). Effect and significance of ITF on PI3K/AKT/GSK-3ßpathway in neonatal rat necrotizing enterocolitis. Chin. J. Clinician. (Electronic Edition) 12: 5392-5396.

Lin HC, Su BH, Chen AC, Lin TW, et al. (2005). Oral probiotics reduce the incidence and severity of necrotizing enterocolitis in very low birth weight infants. Pediatrics 115: 1-4.

Lin HC, Hsu CH, Chen HL, Chung MY, et al. (2008). Oral probiotics prevent necrotizing enterocolitis in very low birth weight preterm infants: a multicenter, randomized, controlled trial. Pediatrics 122: 693-700.

Lodish HF, Zhou B, Liu G and Chen CZ (2008). Micromanagement of the immune system by microRNAs. Nat. Rev. Immunol. 8: 120-130.

Martin M, Rehani K, Jope RS and Michalek SM (2005). Toll-like receptor-mediated cytokine production is differentially regulated by glycogen synthase kinase 3. Nat. Immunol. 6: 777-784.

McElroy SJ, Hobbs S, Kallen M, Tejera N, et al. (2012). Transactivation of EGFR by LPS induces COX-2 expression in enterocytes. PLoS One 7: e38373.

Nahid MA, Satoh M and Chan EK (2011a). Mechanistic role of microRNA-146a in endotoxin-induced differential crossregulation of TLR signaling. J. Immunol. 186: 1723-1734.

Nahid MA, Satoh M and Chan EK (2011b). MicroRNA in TLR signaling and endotoxin tolerance. Cell Mol. Immunol. 8: 388-403.

Pang M, de la Monte SM, Longato L, Tong M, et al. (2009). PPARdelta agonist attenuates alcohol-induced hepatic insulin resistance and improves liver injury and repair. J. Hepatol. 50: 1192-1201.

Rana TM (2007). Illuminating the silence: understanding the structure and function of small RNAs. Nat. Rev. Mol. Cell. Biol. 8: 23-36.

Sodhi CP, Shi XH, Richardson WM, Grant ZS, et al. (2010). Toll-like receptor-4 inhibits enterocyte proliferation via impaired beta-catenin signaling in necrotizing enterocolitis. Gastroenterology 138: 185-196.

Taganov KD, Boldin MP, Chang KJ and Baltimore D (2006). NF-kappaB-dependent induction of microRNA miR-146, an inhibitor targeted to signaling proteins of innate immune responses. Proc. Natl. Acad. Sci. U. S. A. 103: 12481-12486.

Wu D and Pan W (2010). GSK3: a multifaceted kinase in Wnt signaling. Trends Biochem. Sci. 35: 161-168.

Xiong F, Cheng X, Liu FM and Chen SH. (2005). Effects of bifidobacteria on the number of mouse intestinal dendritic cells. Chin. J. Microecol. 17: 405-407. 